Meta

Journal des tradlucteurs

Translators' Journal

\title{
Les mots « intraduisibles »
}

\section{Paul A. Horguelin}

Volume 14, numéro 2, juin 1969

URI : https://id.erudit.org/iderudit/003109ar

DOI : https://doi.org/10.7202/003109ar

Aller au sommaire du numéro

Éditeur(s)

Les Presses de l'Université de Montréal

ISSN

0026-0452 (imprimé)

1492-1421 (numérique)

Découvrir la revue

Citer cet article

Horguelin, P. A. (1969). Les mots « intraduisibles ». Meta, 14(2), 104-106.

https://doi.org/10.7202/003109ar

Ce document est protégé par la loi sur le droit d'auteur. L'utilisation des services d'Érudit (y compris la reproduction) est assujettie à sa politique d'utilisation que vous pouvez consulter en ligne.

https://apropos.erudit.org/fr/usagers/politique-dutilisation/
Cet article est diffusé et préservé par Érudit.

Érudit est un consortium interuniversitaire sans but lucratif composé de l’Université de Montréal, l'Université Laval et l'Université du Québec à Montréal. Il a pour mission la promotion et la valorisation de la recherche. https://www.erudit.org/fr/ 


\section{MOTS "INTRADUISIBLES"}

L'Association des courtiers en valeurs mobilières a publié en 1968 un Lexique des termes employés dans le commerce des valeurs mobilières ${ }^{1}$. On y trouve quelque 200 mots ou expressions propres à ce domaine, avec leur équivalent anglais et une définition. En parcourant ce lexique, nous avons relevé une douzaine de termes que les auteurs ont renoncé à traduire. Peut-être nos lecteurs voudront-ils nous apporter leurs lumières et repousser ainsi les frontières de l'intraduisible.

Cash flow - Il n'existe pas en français de terme qui a exactement la même signification que ce terme anglais. C'est en somme la rentrée au cours d'un exercice de nouvel argent provenant de l'exploitation. On calcule ordinairement le cash flow en faisant l'addition des postes suivants de l'état des profits et pertes: le bénéfice net, les amortissements et, le cas échéant, les impôts différés.

Jitney - Le sens dans lequel ce terme est employé ici en bourse le rend intraduisible. Mais on comprendra mieux son emploi si l'on sait qu'en anglais il sert, entre autres, à désigner un véhicule qui transporte des passagers à un tarif très modique. Un jitney en bourse est un agent de change qui exécute les ordres d'un autre agent de change dont le volume d'affaires n'est pas suffisant pour justifier l'emploi d'un trader pour le représenter sur le parquet de la bourse. Pour s'occuper de l'exécution de ses ordres et de la compensation de ses opérations d'achat et de

1. L'Association a également publié deux brochures: How to Read Financial Statements et Comment lire les états financiers. On peut se les procurer, de même que le lexique, en s'adressant au siège de l'Association (355 ouest, rue Saint-Jacques, Montréal. Téléphone: $842-9034)$. Le prix de chaque publication est de $\$ 1.00$. 
vente, le jitney compte à l'agent de change une commission d'un quart du tarif officiel fixé par la bourse.

Lois dites blue sky - C'est le nom populaire que l'on donne aux lois de divers états et provinces promulguées dans le but de protéger le public contre la fraude en valeurs mobilières. L'expression tire son origine d'un jugement rendu aux États-Unis dans une cause de fraude sur des valeurs mobilières dans lequel le juge déclarait qu'il s'agissait d'une combine spéculative qui n'avait pas plus de fondement que tant de pieds carrés de ciel bleu. Aujourd'hui, lorsqu'une émission de valeurs peut être offerte au public parce qu'elle a été autorisée par la commission de valeurs mobilières concernée, on dit que cette émission a été blue skyed.

Negative pledge - On ne traduit pas ce terme. C'est l'engagement que prend (dans l'acte de fiducie) une compagnie qui émet des débentures de ne pas émettre par la suite d'obligations garanties par hypothèque à moins d'étendre cette garantie hypothécaire à toutes les débentures alors en cours.

Nominee - Ce terme ne se traduit pas. C'est l'organisme ou individu au nom duquel sont enregistrées des valeurs dont cet organisme ou individu est titulaire à titre de fiduciaire pour le compte d'un tiers.

Ordres on stop - Ce sont des ordres à cours limité, mais dans lesquels la fonction de la limite se trouve inversée. Dès que cette limite est atteinte, l'ordre devient un ordre au mieux. Par exemple, si vous détenez des actions dont le cours actuel est de $\$ 50$ et qui vous ont coûté $\$ 35$, vous pouvez protéger votre profit en donnant à votre agent de change un ordre de vente on stop à $\$ 46$. Si le cours baisse à $\$ 46$ votre ordre devient automatiquement un ordre au mieux et vos titres sont vendus immédiatement au meilleur cours possible. On peut aussi donner un ordre d'achat on stop. Vous pouvez par exemple décider que si le cours d'une action, se vendant actuellement à $\$ 25$, venait à atteindre $\$ 28$ il aura traversé un niveau de résistance et pourra monter plus haut. Un ordre d'achat on stop à $\$ 28$ sera exécuté immédiatement au mieux dès que le titre atteindra ce cours.

Put through - Lorsqu'un agent de change a un ordre de vente d'un client pour un titre quelconque et que d'autre part il a un ordre d'achat d'un autre client pour le même titre, les règlements des bourses l'autorisent à faire cette opération sur le parquet de la bourse dans les limites du cours du titre au moment de l'opération et sans l'intervention d'un de ses confrères, sauf pour parapher sa fiche.

Switch, switching - Lorsqu'il s'agit d'opérations sur titres, ces termes ne sont pas traduits et on les emploie comme tels aussi bien en Europe qu'en Amérique du Nord. Il s'agit simplement de la vente de titres que l'on détient pour les remplacer par d'autres qui nous paraissent plus intéressants.

Ticker - C'est ce dispositif qui imprime et transmet par tout le pays les cours et le volume des opérations en bourse dans les quelques minutes qui suivent leur exécution.

Trader et trading - Bien que les équivalents français de ces termes soient négociant et négociation, ceux-ci s'emploient rarement dans le commerce des valeurs mobilières au Canada. On préfère employer les termes anglais qui sont mieux compris et qui ne portent pas à confusion. Traduire ces termes par arbitragiste et arbitrage est une erreur. Voir à ce sujet la définition du terme "arbitrage ». 
Up tick ou plus tick - C'est un terme de bourse qui ne peut se traduire. Le tick est une abréviation de ticker. Il sert à désigner une négociation faite à un cours supérieur à celui de la négociation précédente. Le terme zero-plus tick désigne une opération faite au même cours que la précédente mais que ce cours est différent et supérieur à celui auquel on a traité précédemment. Inversement, les termes down tick ou minus tick et zero-minus tick signifient qu'il s'agit d'une opération à un cours inférieur.

Voting trust - C'est un moyen employé pour confier le contrôle d'une compagnie à certains gérants appelés voting trustees pour une période de temps déterminée ou jusqu'à ce que certaines fins aient été atteintes. Les actionnaires qui déposent leurs actions entre les mains d'un fiduciaire en vertu d'une convention de voting trust, reçoivent en échange des certificats voting trust qui leur rendent les mêmes droits qu'ils possédaient auparavant, sauf le droit de vote.

Traducteurs, à vous de relever le défi !

Paul A. Horguelin 